\title{
Performance Optimization of Forward Aware Factor -Energy Balanced Routing Protocol In Wireless Sensor Networks
}

\author{
Sarika P C, Juby K Baby \\ Department of Electronics and Communication Engineering Vidya A cademy of Science and Technology, \\ Thalakkottukara, Affiliated to Calicut University \\ Department of Electronics and Communication EngineeringHindusthan college of engg \&technology, \\ Coimbatore Anna University Chennai
}

\begin{abstract}
A wireless sensor network (WSN) consists of a large number of wireless sensor nodes. Since wireless sensor nodes are battery powered devices, they have limited processing and transmission power. In order to transmit sensing data to receiver effectively, it is important to design a routing protocol for WSNs. Since energy conservation is a key issue in WSNs, data aggregation should be exploited in order to save energy. In this case, redundant data can be aggregated at intermediate nodes reducing the size and number of exchanged messages and, thus, decreasing communication costs and energy consumption. This paper propose a Forward Aware Factor-Energy Balanced Routing Method (FAF-EBRM). In the experiments, this method is compared with LEACH. Experimental results show that this method FAF-EBRM outperforms LEACH, which balances the energy consumption, prolongs the network function lifetime and provides the best aggregation quality .
\end{abstract}

Keywords: forward aware factor(FAF), Energy balance, Routing, Wireless sensor networks(WSNs).

\section{Introduction}

A Wireless Sensor Networks (WSNs) is a self-organization wireless network system constituted by numbers of micro sensors with limited energy. They are deployed to monitor the sensing field and collect information from physical or environmental condition and to co-operatively pass the collected data through the network to a main location. Due to the limited energy and communication ability of sensor nodes, it is important to design a network topology, routing algorithm and protocol for large-scale WSN communication system. Energy consumption is an important factor in system designs of WSNs. Traditionally, there are two approaches to accomplish the data collection task: Direct communication, and Multi-hop forwarding. In one hop wireless communication, the sensor nodes upload data directly to the sink, which may result in long communication distances and degrade the energy efficiency of sensor nodes. On the other hand, in multi-hop forwarding, data are transferred from the nodes to the sink through multiple relays, and thus communication distance is reduced[7]. However, since nodes closer to the sink have a much heavier forwarding load, their energy may be depleted rapidly, which degrades the network performance.

Clustering is an effective technique to reduce energy consumption in WSNs[13]. In clustering algorithm, a number of nodes in a network will be chosen as the cluster heads (CHs) and the remaining nodes will be regarded as the cluster members(CMs). $\mathrm{CMs}$ will form connections with the $\mathrm{CHs}$. A $\mathrm{CH}$ will collect data from its CMs. In WSN clustered hierarchical routing protocols, sometimes CMs are closer to the sink than $\mathrm{CH}$, but it should transmit data to $\mathrm{CH}$ first. This backward transmission result in waste of energy.

The aim of this project is to propose a new energy-balance routing protocol that uses forward transmission area(FTA) based on position of sink and final data flow direction. In other words, FTA define forward energy density which constitutes forward-aware factor with link weight, and propose a new communication protocol based on forward-aware factor,thus balancing the energy consumption and prolonging the network function lifetime[14],[16]

\section{Related Work}

\subsection{Low Energy Adaptive Clustering Hierarchy(LEACH) Protocol}

LEACH protocol is one of the most famous hierarchical routingprotocol for wireless sensor networks to increase the life time of network. In LEACH, all the nodes in a network organize themselves into local clusters. The protocol is divided into a setup phase when the clusters are organized and a steady state phase when $\mathrm{CH}$ receive data from all the $\mathrm{CMs}$, perform data aggregationand transmit data to the remote base station. The operation of LEACH is illustrated in Fig1. In clustering algorithm, $\mathrm{CH}$ node is much more energy-intensive than a CM. Thus, when a $\mathrm{CH}$ node in a cluster dies, all the CMs inside that cluster lose communication ability.

LEACH allows randomized rotation of the high-energy cluster-head position such that it rotates among the sensors. In this way, the energy load associated with being a $\mathrm{CH}$ is evenly distributed among the sensing nodes. The CH node will know all the CMs in the cluster. Therefore it can create a TDMA schedule that informs 
each node exactly when to transmit its collected data

In the setup phase, each sensor node chooses a random number, $\mathrm{r}$, between 0 and 1 . If this random number is less than a certain threshold value, $\mathrm{T}(\mathrm{n})$, the node becomes a $\mathrm{CH}$ for the current round.

Threshold value $\mathrm{T}(\mathrm{n})$ is calculated by the equation,

$$
\begin{array}{ll}
T(n)=p /(1-p \times r \bmod (1 / p)) & , n € G \\
0 & \text { else }
\end{array}
$$

This equation incorporates the desired percentage to become a $\mathrm{CH}$, the current round, and the set of nodes that have not been selected as a $\mathrm{CH}$ in the last $(1 / \mathrm{P})$ rounds where $\mathrm{p}$ is cluster head probability. If random number is less than $\mathrm{T}(\mathrm{n})$, the node will broadcasts an advertisement message (ADV) as the $\mathrm{CH}$. This message contains the node's ID and a header that distinguishes this message as an announcement message. Each noncluster-head selects the $\mathrm{CH}$ that requires the minimum communication energy, based on the received signal strength of the advertisement from each $\mathrm{CH}$. After the selection of $\mathrm{CH}$, each node must inform thecluster-head node that it will be a member of the cluster. Each node sends a join-request message (Join-REQ) back to the selected $\mathrm{CH}[2],[6]$.

In the steady-state phase, operation is broken into frames where nodes transmit their data to the $\mathrm{CH}$ at most once per frame during their allocated transmission slot. To reduce energy dissipation, each CM sets the amount of transmission power by using power control. It is based on the received strength of the cluster-head advertisement. The $\mathrm{CH}$ receives all the data from the nodes in the cluster by keeping its receiver on and then the resultant data are sent from the $\mathrm{CH}$ to the base station.

\section{Forward Aware Factor -Energy Balanced Routing Method(FAF-EBRM)}

In WSN clustered hierarchical routing protocol, sometimes cluster members in a cluster are nearer to the sink than the $\mathrm{CH}$, but it should transmit data to $\mathrm{CH}$ first. It results backward transmission of data and thus leads to waste of energy.

In this method, an energy-balanced routing protocol is designed that uses forward transmission area(FTA) based on position of sink and final data flow direction. In other words, FTA define forward energy density which constitutes forward-aware factor with link weight, and propose a new communication protocol based on forward-aware factor, thus balancing the energy consumption and prolonging the network function lifetime[3].

\subsection{Description of Network model}

Consider a rectangular sensing field $\mathrm{W} \times \mathrm{H}$ in which sensor nodes are randomly distributed as shown in Fig 4.1. Regional central node( cluster head) receives data from all the CMs and then transfers tothe sink node(sink).

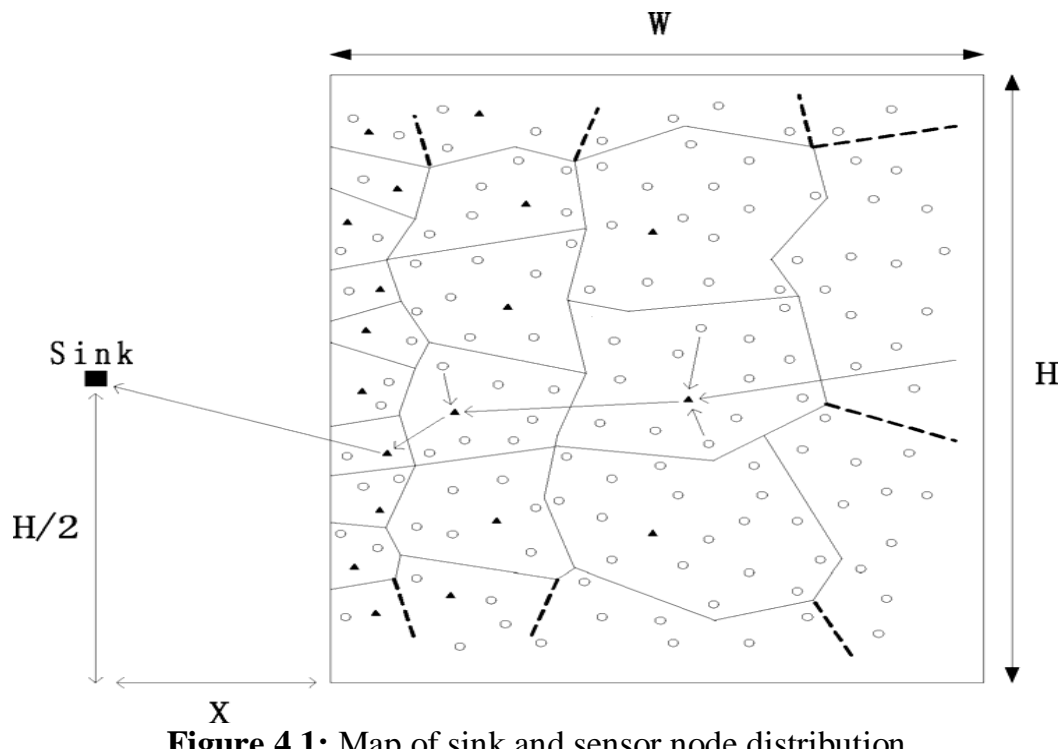

The descriptions of network model are given below

1) All sensor nodes are isomorphic and they have limited energy and communication ability. Here, identifier for a node is represented as ' $\mathrm{i}$ '. 
2) $\mathrm{CH}$ is more energy intensive than a $\mathrm{CM}$. Thus, when a $\mathrm{CH}$ in a cluster dies, all the $\mathrm{CMs}$ inside that cluster lose communication ability. However, addition of energy of sink node is possible. But, it is not possible to change the location of nodes and sink after being fixed.

3) According to distance to receiver, nodes can vary its transmission power. The sink node can broadcast advertisement message to all nodes in the sensing field. The distance between source and receiver can be determined by received signal strength. When the data transmission distance is more than certain threshold $\mathrm{d} 0$, the energy consumption would rise sharply. The threshold value is given by,

$$
\mathrm{d}_{0}=\sqrt{\frac{\varepsilon_{\mathrm{fs}}^{2}}{\varepsilon_{\mathrm{mp}}}}
$$

where, $\varepsilon_{\mathrm{fs}}$ and $\varepsilon_{\mathrm{mp}}$ are the energy coefficients.

\subsection{Establishment of the Network}

Let FTA(i) be the forward transmission area of node(i), N(i) be the set of nodes that have communication link with node (i), N'(i) be the set of nodes of $N(i)$ that have edge with node $i, d_{i j}$ be the distance between node $\mathrm{i}$ and node $\mathrm{j}$. Consider a circle $\odot_{1}$ with sink as the centre and another circle $\odot_{2}$ with node, $i$ as the centre and $d_{i p}$ as the radius as shown in Fig 4.2

$$
\begin{aligned}
& \text { FTA(i) }=\odot \mathrm{O}_{1} \cap \odot \mathrm{O}_{2} \\
& \text { ij } \\
& \text { d } \\
& \mathrm{d}_{\mathrm{ip}}=\max
\end{aligned}
$$

This paper proposes an energy balanced routing protocol that uses forward transmission area (FTA) based on position of sink and final data flow direction. In fig 4.2, the arc of circle $\odot_{1}$ shows the possibility of backward transmission of node i. Circle $\odot_{2}$ contains all nodes that directly connected with node i. These 2 circles contains all possible next nodes under topology and routing algorithm.FTA(i) is the overlap section of these 2 circles.

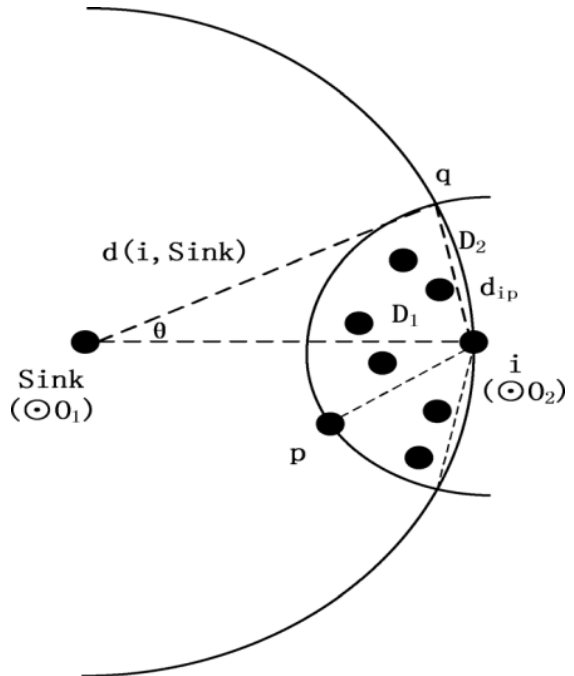

Figure 4.2: Forward Transmission Area of node $\mathrm{i}$

By using (4), the area of FTA(i) is $\mathrm{S}_{\mathrm{FTA}}$ (i) and can be written as

$$
\begin{gathered}
\operatorname{maxd} \\
\max \left(d_{i j}\right) \\
2 / 3 \prod-\frac{\sqrt{3}}{2}
\end{gathered}
$$

Let $d(i, \operatorname{sink})=d_{1}, d_{i p}=\max \left(d_{i j}=d_{2}\right.$. According to cosine theorem, angle $\theta$ can be written as

$$
\begin{gathered}
1-\frac{1}{2} \\
\theta=\operatorname{arcos}
\end{gathered}
$$

Consider a case in which sink is a neighbour of node $i$ as shown in Fig 4.3. In this case, $d_{1}$ become equal to $d_{2}$. 
If $\mathrm{d}_{1}=\mathrm{d}_{2}$, then minimum area of FTA(i) becomes $\left(\frac{2 \Pi}{3}-\frac{\sqrt{3}}{2} \mathrm{~d}_{2}^{2}\right.$

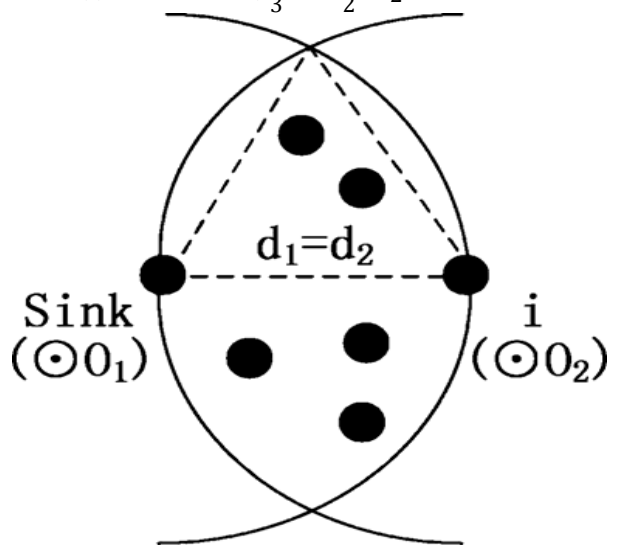

Figure 4.3: Minimum FTA of node i

Consider an another case in which $\mathrm{d}_{1}$ tends to infinity as shown in Fig 4.4.In this case, the maximum area of FTA(i) is $\prod d_{2}^{2} / 2$. When the radius of $\odot_{1}$ become infinity, its arc tends to be a straight line. This arc passes the centre of $\odot_{2}$ and approximately dividing $\odot_{2}$ equally.

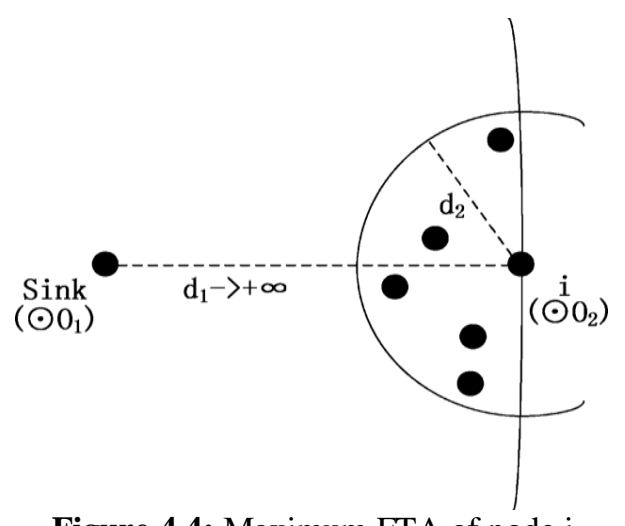

Figure 4.4: Maximum FTA of node i

The area of FTA(i) satisfies the condition of inequality

$$
0<\mathrm{S}_{\mathrm{FTA}}(\mathrm{i})<\frac{1}{2} \Pi \mathrm{d}_{0}^{2}
$$

Where $\mathrm{d}_{0}$ is the communication radius. The node's forward energy density FED(i,t) satisfies the condition of equality.

$$
\mathrm{FED}(\mathrm{i}, \mathrm{t})=\frac{\sum_{\mathrm{jeFTA}(\mathrm{i})} \mathrm{E}_{\mathrm{j}}(\mathrm{t})}{\mathrm{S}_{\mathrm{FTA}}(\mathrm{i})}
$$

Where $E_{j}(t)$ is the energy of node jat time $t$ and the numerator in (8) represents energy of all the neighbour combined in function FTA(i). The forward aware factor (FAF) of the transmission link between node $i$ and node $\mathrm{j}$ can be written as

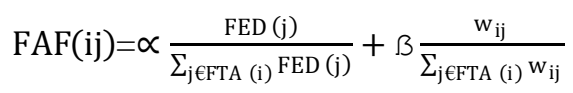

In the above equation, the first term represents the FED of all possible next hop nodes that means the ability to transmit data. The second term represents the weight of transmit link which is used to select next hop node directly. In (9), the denominator of the first term represents FAF of all the neighbours combined in FTA(i) and that of second term represents all of the link weights combined that $i$ has in FTA. $\propto$ and $\beta$ are positive harmonic coefficients and they are related by

$$
\propto+\beta=1
$$




\subsection{Design of the Enhanced Forward Aware Factor -Energy Balanced Routing Method (FAF-EBRM)}

Large scale WSN uses FAF-EBRM for static data collection and event detection[1]. The routing algorithm of this method is given below.

1) Determine FTA(i) and all of the possible next-hop nodes of node i . First, take as the communication radius, determine the set of all of the nodes that have edges with i, N'(i). Select the nodes that closer to Sink than i does, which constitute the set of all of the possible next-hop nodes and the furthest node determine FTA(i).

2) Determine FTA(j)and $S_{\text {FTA }}(j)$ of each possible next-hopnode. Determine FTA(j) as we determined FTA(i) .

3) Calculate FED(j) of each possible next-hop node according to (8).

4) Calculate the weight of edges between $i$ and each nodes.

5) Calculate FAF of each possible transmit link by using (9). Choose the next-hop node using the following equation

$$
\mathrm{j}=\max _{\mathrm{j}}[\mathrm{FAF}(\mathrm{ij})]
$$

6) If there is no node closer to Sink than $i$ in $N^{\prime}(i)$, directly compare FAF of all of the nodes in N'(i), and choose the next-hop node according to (10). If there is no node in $N^{\prime}(i)$, i will increase the transmit power to get a longer radius than $\mathrm{d}_{0}$ until connected with another node, or $\mathrm{i}$ will abandon the packet.

7) If Sink is among the forward transmit nodes, i will transmit data directly to Sink and accomplish the procedure[11].

For energy efficient transmission in event-driven WSN, Data should be reduced. It requires proper routing method for reliable transmission of aggregated data to sink from the source nodes. This paper propose a new communication protocol based on forward-aware factor in order to determine next-hop node to reduce the number of transmissions and thus balancing the energy consumption, prolonging the network function lifetime and to improve QoS of WSN.

\section{Simulation Results}

Here, we compare LEACH and FAF-EBRM by three parameters: energy-balanced factor (EBF), number of last- surviving nodes (NLN) and function lifetime (FL), packets reception radio (PRR). To measure the balance of energy consumption of routing protocols, EBF is defined as the standard deviation of all the nodes' residual energy

$$
\begin{aligned}
& \frac{1}{N} \\
& \mathrm{E}_{\mathrm{i}}
\end{aligned}
$$

$$
\mathrm{EBF}=\sqrt{ }
$$

Where $\mathrm{N}$ is the number of the whole network nodes, $\mathrm{E}_{\mathrm{i}}(\mathrm{t})$ is the residual energy of node at time and $\mathrm{E}_{\text {avg }}(\mathrm{t})$ is the average value of the residual energy of all of the nodes.

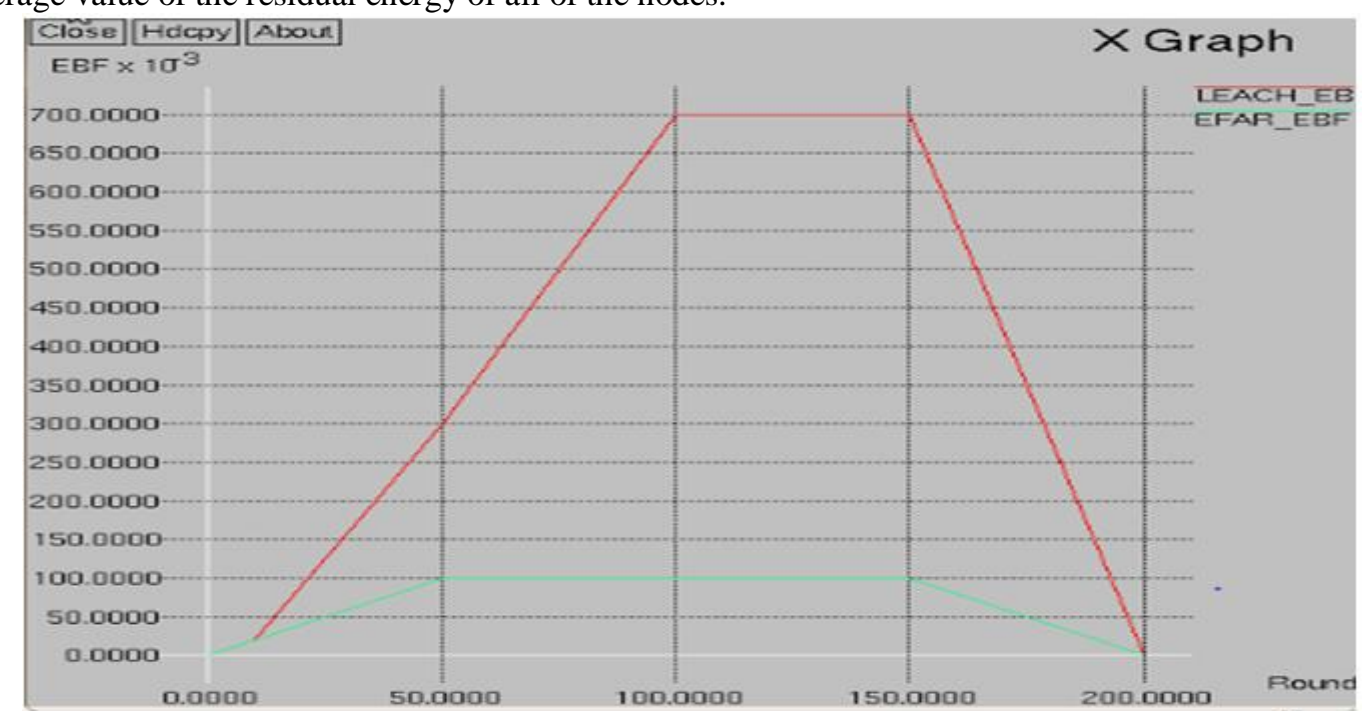

Figure 6.1: Simulation showing comparison of Energy Balanced Factor (EBF)

FL is directly related to NLN; the definition and requirement of FL is different under different conditions, as some require no death node, and some can still work when a certain percentage of nodes still work. In our experiment, we consider two conditions of FL, one is the time from the network begins to the first death of the nodes, another is the time from the network begin to half the nodes dead. 


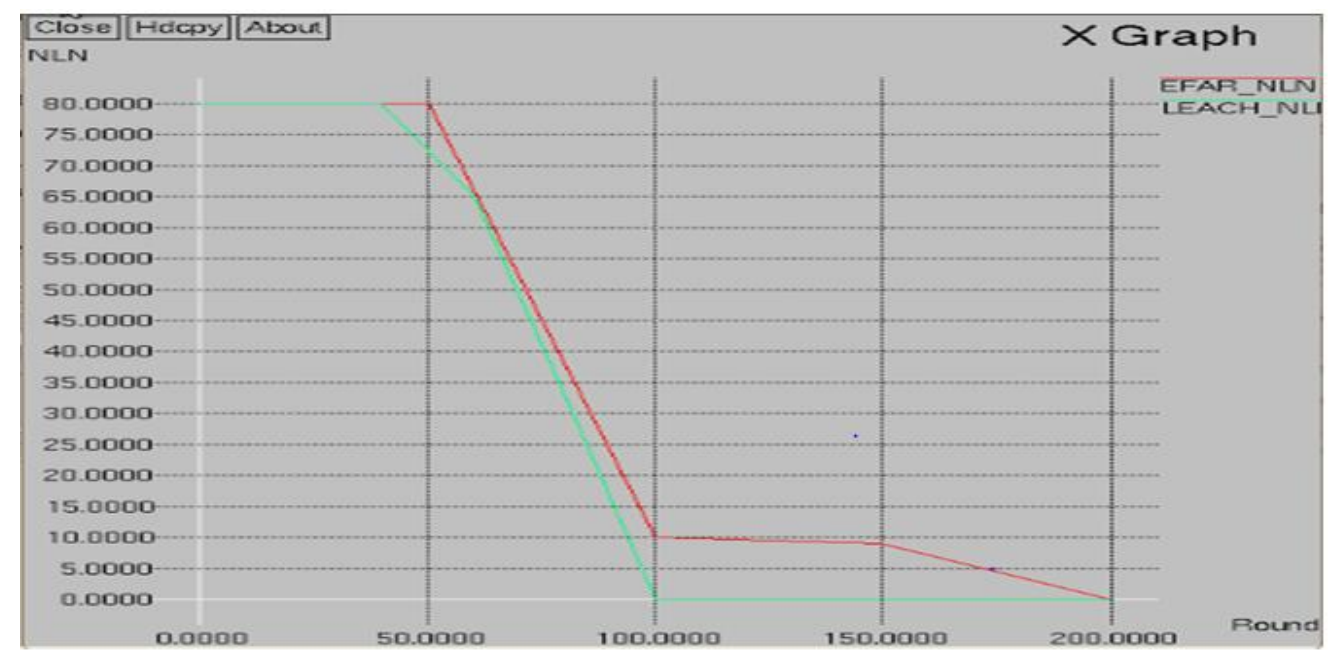

Figure 6.2: Simulation showing comparison of Number of Last-surviving Nodes (NLN)

PRR means the ratio of the data that sink actually received to the data that sink is supposed to be received. PRR can measure WSN work situation intuitively.

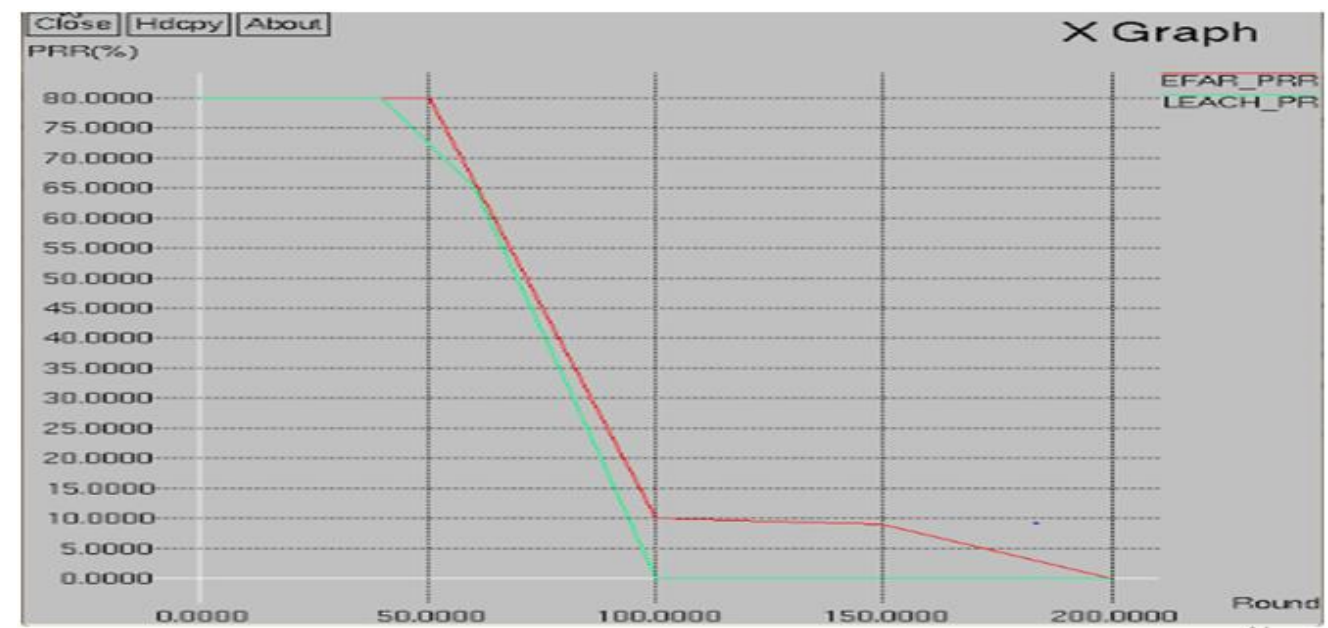

Figure 6.3: Simulation showing comparison of Packet Reception Ratio(PRR in \%)

To compare two protocols conveniently and intuitively,EFAF-EBRM also uses round as the time scale. Figs. 6-8 shows that the EBF, NLN, and PRR of three protocols in 200 rounds experiments. In Fig. 6, the EBF of FAF-EBRM increase slightly at first and keep a stable situation, then increase a little time, and return to 0 as the energy of the whole network is using up. In Fig. 7, the first death of FAF-EBRM node turns up until Round 200 , and the procedure of the nodes' death is fast and late. In Fig. 8, the PRR of FAF-EBRM keeps 100\% ratio for 200 rounds, and the decline stage accounting for a small proportion.

\section{Conclusion And Future Work}

Energy balanced routing algorithms play an important role in event-based WSNs. In this work, we presented the FAF EBRM ( Forward Aware Factor and Data Agregation) algorithm. Our FAF-EBRM algorithm was extensively compared to other known routing algorithms, LEACH, regarding scalability, communication costs, delivery efficiency, aggregation rate, and aggregated data delivery rate. By maximizing the aggregation points and offering a fault tolerant mechanism to improve delivery rate, the obtained results clearly show that FAF-EBRM outperformed LEACH algorithms for all evaluated scenarios. Also, we show that our proposed algorithm has some key aspects required by WSNs aggregation aware routing algorithms such as a reduced number of messages for setting up a routing tree, maximized number of overlapping routes, high aggregation rate, and reliable data aggregation and transmission. As future work, spatial and temporal correlation of the aggregated data will also be taken into consideration as well as the construction of a routing tree that meets application needs. We also plan to modify the Proposed algorithm to stochastically select nodes that will be part of the communication structure. The goal is to find a balance between the overhead and the quality of the routing tree. In addition, new strategies will be devised to control the waiting time for aggregator 
nodes based on two criteria: average distance of the event coordinators, and spatial and semantics event correlation.

\section{Acknowledgment}

Prof. Dr S.Swapna Kumar, HOD of the Electronics and Communication Engineering department who contributed enormously to this paper and my research. His support during my work was incredibly invauable. I would like to thank Ms.Jubi K. Baby who provided a precious support to my work with her careful and knowledge able revision of the manuscript.

\section{References}

[1]. Abd El-kader S M and Eissa H S, Mohammed M. Zahra (2011) 'An Optimized Energy-aware Routing Protocol for Wireless Sensor Network Egyptian Informatics Journal' vol 34 no 12 pp.61-72

[2]. Abusaimeh H and Yang H (2009) 'Dynamic Cluster Head for Lifetime Efficiency in WSN International Journal of Automation and Computing' vol 67 no 4 pp.48-54.

[3]. Attea B A and Khalil E A (2011) 'Energy-aware evolutionary routing protocol for dynamic clustering of wireless sensor networks' vol 122 no.1 pp.104-124

[4]. Awan I and Bajaber T (2011) 'Adaptive decentralized re-clustering protocol for wireless sensor Networks : Journal of Computer and System Sciences' vol 77 no 2 pp. 282-292

[5]. Balakrishnan H, Chandrakasan A, Heinzelman W R(2000) 'Energy Efficient Communication Protocol for Wireless Microsensor Networks. IEEE. Published in the Proceedings of the Hawaii International Conference on System Sciences' vol 81 no 1 pp.41-47.

[6]. Boavida F, Camilo C, Carreto J and Silva S (2006) ' An Energy-Efficient Ant-Based Routing Algorithm for Wireless Sensor Networks' vol 35 no 9 pp.879-885

[7]. Chakraborty A, Chakraborty K, Mitra S K and M.K. Naskar (2009) 'An Optimized Lifetime Enhancement Scheme for Data Gathering in Wireless Sensor Networks' vol 56 no 8 pp.554-560.

[8]. Chen Y, Liu X and Zhang D (2012) 'Improvement of LEACH in Wireless Sensor Networks Based on Balanced Energy Strategy' vol 44 no 27 pp.1478-1486

[9]. Cho Y, Heo J and Hong J (2007) ' PEACH: Power-efficient and adaptive clustering hierarchy protocol for wireless sensor networks' vol 30 no 7 pp. 2842-2852

[10]. Defago X, Nguyen L T, and Shinoda Y (2008) ‘An Energy Efficient Routing Scheme for Mobile Wireless Sensor Networks’ vol 88 no.6 pp.234-241

[11]. Doerner K, Gutjahr W J and Hartl R F (2001) 'Ant Colony Optimization in Multiobjective Portfolio Selection, Citeseer - 4th Metaheuristics International Conference'vol 34 no 11 pp.345-354

[12]. Erdogan A T and Zhao J (2006) 'A Novel Self-organizing Hybrid Network Protocol for Wireless Sensor Networks Proceedings of the First NASA/ESA Conference on Adaptive Hardware and Systems' vol 67 no 9 pp.542-549

[13]. Eu T, Seah K G and Tan H P (2009) 'Routing and Relay Node Placement in Wireless Sensor Networks Powered by Ambient Energy Harvesting' vol 45 no 32 pp.1345-1352

[14]. Fahmy S and Younis O (2004) 'HEED: A Hybrid, Energy-Efficient, Distributed Clustering Approach for Ad-hoc Sensor Networks' vol 53 no 9 pp.234-245

[15]. Giel A T, Pesch D and Wenning B L (2010) 'Environmental monitoring aware routing: making environmental sensor networks more robust, Carmelita Gorg In: Telecommunication Systems' vol 43 no 1 pp. 3-11.

[16]. Grawal D.P and Manjeshwar A (2001) 'A Routing Protocol for Enhanced Efficiency in Wireless Sensor Networks' vol 43 no 5 pp.479-486

[17]. Jiang T, Zang W and Zhao C (2010) ‘ An Energy Consumption Optimized Clustering Algorithm for Radar Sensor Networks Based on an Ant Colony Algorithm, Hindawi Publishing Corporation' vol 25 no 34 pp.2322-2327

[18]. Krishnamachari B and Wicker S (2002) 'Modeling data-centric routing in wireless sensor networks. In: Proceedings of the IEEE INFOCOM' vol 29 no 35 pp.267-273

[19]. Kumar D, Patel R B and Trilok G (2009) 'EEHC: Energy efficient heterogeneous clustered scheme for wireless sensor networks Computer Communications' vol 566 no 23 pp.256-667

[20]. Lindsey S and Raghavendra C S (2001) 'PEGASIS: Power-Efficient GAthering in Sensor Information Systems' vol 45 no 7 pp.1678-1687

[21]. Luz Y M and Wong S (2007) 'An energy-efficient multipath routing protocol for wireless sensor networks, International journal of communication systems' vol 34 no 2 pp.747-766

[22]. Meenaxi S and Singh R (2013) 'An Optimized Low Loss Energy-Aware Routing Protocol for Wireless Sensor Networks International Journal of Computer Applications' vol 65 no 21 pp 345-351

[23]. Meenaxi T and Verma P (2013) 'Compartive analysis of QoS parameters in wireless sensor networks using ant colony optimization. International Conference on Control, Communication and Computer Technology’ vol 17 no 45 pp. 1132-1139

[24]. Misra S , Obaidatc R and Verma K (2010) 'An ant swarm-inspired energy-aware routing protocol for wireless ad-hoc networks. The Journal of Systems and Software' vol 3 no 38 pp. 2188-2199

[25]. Nayebi A and Sarbazi-Azad J (2011) 'Performance modeling of the LEACH protocol for mobile wireless sensor networks' vol 24 no.26 pp.812-821

[26]. Qi X and Zheng G (2012) 'Clustering routing algorithm of wireless sensor networks based on Bayesian game Journal of Systems Engineering and Electronics' vol 23 no 1 pp.154-159

[27]. Rabaey J M and Shah R C (2002) 'Energy Aware Routing for Low Energy Ad Hoc Sensor Networks IEEE wireless Communications and Networking Conf.' vol 78 no 4 pp 936-942

[28]. Song M and Youssef K (2011) 'Unequal clustering algorithm for WSN based on fuzzy logic and improved ACO' vol 18 no 6 pp.89-97

[29]. Tafazolli R and Vural S (2011) 'An Energy-efficient Clustering Solution for Wireless Sensor Networks IEEE transactions on wireless communications' vol 9 no 18 pp.561-568

[30]. Xue L, Yanjing S and Yanjun H (2011) 'An energy efficiency clustering routing protocol for WSNs in confined area' vol 4 no 9 pp.246-251

[31]. Younis M and Youssef M (2002) 'Energy-Aware Routing in Cluster-Based Sensor Networks' vol 3 no 3 pp. 437-444 